\title{
Bilateral involvement by age related maculopathy lesions in a population
}

\author{
Jie Jin Wang, Paul Mitchell, Wayne Smith, Robert G Cumming
}

\begin{abstract}
Aims-To describe the influences of age and sex on the frequency of bilateral age related macular degeneration (AMD) and age related maculopathy (ARM) lesions. Methods-The Blue Mountains Eye Study examined 3654 older Australians, 82\% of permanent residents living in an area west of Sydney. Stereo macular photographs were graded for AMD (neovascular maculopathy and geographic atrophy) and early ARM lesions (soft drusen, reticular drusen, hyperpigmentation, and hypopigmentation).
\end{abstract}

Results-Among 230 gradable cases of AMD or early ARM, 183 (80\%) were bilateral. For AMD, $39 / 69$ cases (57\%) were bilateral, while for early ARM, 123/161 cases $(77 \%)$ had signs in both eyes. Of the individual lesions, reticular drusen $(91 \%)$ and indistinct soft drusen $(\mathbf{7 9 \%})$ were most frequently present in both eyes. Geographic atrophy was bilateral in $56 \%$, neovascular AMD in $40 \%$, and distinct soft drusen in $47 \%$, while hyperpigmentation was bilateral in $38 \%$ and hypopigmentation in only $28 \%$ of cases. A consistent age related increase in bilateral distribution was observed for most lesions. After adjusting for effects of age, current smoking, and AMD family history AMD and ARM component lesions, except for soft drusen, were more frequently bilateral in women. This sex difference was significant only for neovascular AMD, odds ratio 7.7 (95\% confidence intervals $1.3-46.7)$. An AMD family history was more frequently reported in cases with bilateral involvement.

Conclusions-This study has documented differences in the age related bilaterality of individual ARM components with higher bilateral rates for reticular or indistinct soft drusen compared with other lesions. The increased bilaterality of most ARM lesions among women is likely to contribute to the increased age adjusted risk of AMD blindness found in women.

(Br F Ophthalmol 1998;82:743-747)

Age related maculopathy (ARM) is the leading cause of blindness in Australia ${ }^{12}$ and in most Western countries. ${ }^{3-5}$ This is mainly attributed to bilateral involvement of the disease, with frequent second eye involvement from the two alternate late stages with increasing age, with most cases not able to be helped by treatment. Recently, an international group of ophthalmologists has agreed upon common terms to describe the lesions of ARM. ${ }^{6}$ The early stages of ARM include soft (large) drusen and hyperpigmentation or hypopigmentation of the retinal pigment epithelium (RPE). Late stages of ARM are termed age related macular degeneration (AMD), a term which includes geographic atrophy ("dry" AMD) and neovascular AMD ("wet", or "exudative", or "disciform” AMD).

Bilateral distribution of the late lesions of age related maculopathy has long been recognised, and the frequency of second eye involvement has been documented in a number of retrospective and prospective case series. ${ }^{7-11} \mathrm{~A}$ study of the symmetry of disciform scars found a significant correlation between eyes in terms of the final scar size, and large macular scars were more frequent in the second eye if the first eye had a large scar. ${ }^{12}$ Bilateral confluent macular drusen have been identified as indicating a higher than average risk of AMD ${ }^{13}{ }^{14}$ Factors which might alter the incidence of second eye involvement have also been explored in a clinic case series. ${ }^{15}$

The quadrantic macular distribution pattern of early ARM lesions were assessed in the Beaver Dam Eye Study. ${ }^{16}$ Interestingly, this study found different distribution patterns for indistinct soft drusen and pigmentary abnormalities among the four quadrants of macular area (superior, temporal, inferior, and nasal). However, neither the Beaver Dam Eye Study nor any other population based studies have described the bilateral distribution in detail of individual AMD and ARM component lesions. Such data are useful and relevant as the impact of bilateral involvement by AMD on quality of life is much greater than from unilateral involvement. Accurate population based data on bilateral involvement may allow estimates of the future cost of AMD. Aims of the current study, therefore, were to describe the bilaterality of AMD and ARM lesions in a defined older population and to document effects of age and sex on the pattern of bilateral involvement, after adjusting for the principal identified risk factors for ARM. ${ }^{17-20}$

Materials and methods

STUDY POPULATION

The Blue Mountains Eye Study is a population based survey of vision and common eye diseases in an urban elderly population (aged 49 years and older) comprising two postcode areas in the Blue Mountains region, west of Sydney, Australia. Details of the survey method and procedures have been previously described. $^{21}$ Of 4433 eligible residents, 3654 (82.4\%) were examined during 1992-4. Family 
history of AMD was assessed using an interviewer administered questionnaire, which included the following questions: "We want to know if eye disease runs in families. Have either of your parents, or any brothers or sisters or children had macular degeneration diagnosed?" An AMD family history was defined from a positive answer to this question; subjects with missing family history data were excluded from analyses. Smoking status was defined as "current" if the participant currently smoked or had stopped smoking for less than 1 year before the examination. Subjects who had ever smoked but had stopped for more than 1 year were defined as "past" smokers.

All participants underwent a detailed eye examination. This included Zeiss FF3 $30^{\circ}$ stereo photographs (Carl Zeiss, Oberkochen, Germany) of Diabetic Retinopathy Study fields one (disc) and two (macula), with non-stereo photographs of fields three (temporal to macula), four and five (upper and lower vascular arcades) and a field nasal to the optic disc. $^{22}$

Two graders performed a masked grading of the stereo fundus photographs to assess presence of AMD or ARM lesions. The Wisconsin Age Related Maculopathy Grading System $^{23}$ was closely followed with minor modifications. ${ }^{21}$ A transparent grid, supplied by Dr Ronald Klein, University of Wisconsin, Madison, including three concentric circles, was placed over one of the stereo photos of field two for each eye. This divided the macular area into three zones: central, inner, and outer circles, corresponding to a radius of 500 , 1500 , and $3000 \mu \mathrm{m}$ from the foveolar centre. All photographs were graded initially by either one of the two graders and all advanced cases were assessed by PM, who also adjudicated any queries or discrepancies. Intergrader and intragrader reliability, assessed on a random subsample of gradable eyes, showed good agreement for all lesions. ${ }^{21}$ In the assessment of lesion bilaterality, all cases with ARM lesions had a detailed regrading by the two graders.

GRADING DEFINITIONS

Age related macular degeneration (AMD) included the two end stage lesions, neovascular maculopathy and geographic atrophy involving the foveal centre. Neovascular lesions included serous or haemorrhagic detachment of the $\mathrm{RPE}$ or sensory retina, or subretinal fibrous tissue. Geographic atrophy was defined as a discrete area of retinal depigmentation at least $175 \mu \mathrm{m}$ in diameter, characterised by a sharp border and the presence of visible choroidal vessels. ${ }^{621}$ Eyes with minimal subretinal fibrosis and widespread surrounding atrophy were classified as having both neovascular AMD and geographic atrophy for the assessment of bilaterality. Cases with neovascular AMD in one eye and geographic atrophy in the fellow eye were graded as unilateral for both neovascular AMD and geographic atrophy, but bilateral for "any AMD".

Early ARM was defined as the absence of AMD and either (1) indistinct soft or reticular drusen or (2) both distinct soft drusen and retinal pigmentary abnormalities, within the area of the grid. ${ }^{21}$ Hyperpigmentation was defined as definite clumps of dark brown or dark green pigment beneath the retina. Hypopigmentation was defined as discrete areas of retinal depigmentation without visible choroidal vessels, often with adjacent pigment clumping. We included cases only in which large drusen (maximum size larger than 125 $\mu \mathrm{m}$ diameter) were present in at least one eye. Subjects with AMD in either eye were excluded from the assessment of bilateral involvement by early ARM lesions.

We examined the following lesion groups for bilaterality:

(1) Any ARM-either AMD or early ARM present in both eyes, including subjects with AMD in one eye and early ARM in the fellow eye

(2) Any $A M D$

(a) neovascular AMD

(b) geographic atrophy

(3) Any early ARM-early ARM in one or both eyes without AMD in either eye

(a) reticular drusen

(b) indistinct soft drusen

(c) distinct soft drusen

(d) retinal pigmentary abnormalities (hyper- or hypopigmentation)

(e) hyperpigmentation

(f) hypopigmentation

(4) Symmetrical soft drusen characteristics

(a) type: at least one soft drusen type in common between the eyes

(b) size: maximum drusen size no more than one grading step different between the two eyes

(c) location: majority of drusen located either within or outside the grid for the two eyes

(d) area: soft drusen area within the central and inner circles no more than one grading step different between the two eyes.

Cases with data available for only one eye were excluded from this study (three AMD cases and 10 early ARM cases, because of coexisting dense cataract, corneal, or retinal lesions).

DATA HANDLING AND STATISTICAL METHODS

Statistical Analysis System (version 6.11) (SAS Institute Inc, Cary, NC, USA) was used for statistical analyses including $\chi^{2}$ statistic, Mantel-Haenszel $\chi^{2}$ trend statistic and logistic regression analyses. In the logistic regression analyses, bilateral (compared with unilateral) involvement by individual ARM lesions or lesion groups, were the dependent variables and were assessed separately in different models. Age was used continuously while sex, current smoking, and AMD family history were used as dichotomous variables. When comparing current with never smokers, past smokers were excluded. Magnitudes of association were expressed as odds ratios (OR) with 95\% confidence intervals (CI). All participants who had ARM lesions and both eyes gradable were included in this study. No statistical adjustment has been made for multiple comparisons. 
Table 1 Bilaterality of selected age related macular degeneration (AMD) and age related maculopathy (ARM) lesions

\begin{tabular}{lrr}
\hline Lesions & $\begin{array}{l}\text { No of } \\
\text { cases }\end{array}$ & $\begin{array}{l}\text { No (\%) with } \\
\text { bilateral } \\
\text { involvement }\end{array}$ \\
\hline Any ARM (AMD + early ARM) & 230 & $183(80)$ \\
Any AMD & 69 & $39(57)$ \\
$\quad$ neovascular AMD & 48 & $19(40)$ \\
geographic atrophy & 39 & $22(56)$ \\
Any early ARM & 161 & $123(76)$ \\
$\quad$ reticular drusen & 35 & $32(91)$ \\
indistinct soft drusen & 118 & $93(79)$ \\
distinct soft drusen & 184 & $87(47)$ \\
pigmentary abnormality & 374 & $140(37)$ \\
hyperpigmentation & 368 & $138(38)$ \\
$\quad$ hypopigmentation & 262 & $73(28)$ \\
\hline
\end{tabular}

\section{Results}

BILATERAL INVOLVEMENT OF ANY ARM, AMD, AND EARLY ARM

After excluding subjects who could not be graded for bilaterality, 230 subjects had any ARM (AMD or early ARM), of whom 183 $(80 \%)$ were bilateral. Taking the two subgroups separately, 69 cases had AMD, including 39 (57\%) who had bilateral AMD lesions, 161 cases had early ARM, including 123 (76\%) who had bilateral lesions. In 20 of the 30 unilateral AMD cases, early ARM was present in the fellow eye. Among people with AMD, 19 of the $48(40 \%)$ neovascular AMD cases were bilateral and 22 of the 39 (56\%) geographic atrophy cases were bilateral (Table 1). In seven of the 29 cases (24\%) with unilateral neovascular AMD, geographic atrophy was present in the fellow eye, while eight of the 17 unilateral geographic atrophy cases (53\%) had neovascular AMD in the fellow eye. Seven cases had coexisting neovascular AMD and GA in the same eye.

For individual early ARM lesions graded, reticular drusen (91\%) and indistinct soft drusen $(79 \%)$ had the highest frequency of bilateral involvement. Distinct soft drusen were bilateral in $47 \%$ and retinal pigmentary abnormalities in $37 \%$ of cases (Table 1). Signs of hyperpigmentation and hypopigmentation were bilateral in $38 \%$ and $28 \%$ of cases, respectively. Graded soft drusen characteristics (type, size, location, and area covered) were also symmetrical between the two eyes in around $60 \%$ of cases (results not shown).

Table 2 Effect of age on bilateral involvement by lesions of age related macular degeneration (AMD) and early age related maculopathy (ARM)

\begin{tabular}{|c|c|c|c|c|c|c|}
\hline \multirow[b]{2}{*}{ Outcomes } & \multirow{2}{*}{$\begin{array}{l}\text { Total no } \\
\text { of cases }\end{array}$} & \multicolumn{4}{|c|}{ Percentage with bilateral involvement } & \multirow{2}{*}{$\begin{array}{l}\text { Mantel } \\
\text { Haenszel } \chi^{2} \\
\text { for trend }\end{array}$} \\
\hline & & age $<60$ & age 60-69 & age $70-79$ & age $\geqslant 80$ & \\
\hline Any ARM & 230 & 43 & 61 & 82 & 90 & $<0.001$ \\
\hline Any AMD & 69 & 0 & 17 & 44 & 71 & 0.003 \\
\hline neovascular AMD & 48 & 0 & 17 & 25 & 54 & 0.03 \\
\hline geographic atrophy & 39 & 0 & 0 & 62 & 56 & 0.78 \\
\hline Any early ARM & 161 & 43 & 59 & 83 & 88 & 0.001 \\
\hline reticular drusen & 35 & 0 & 100 & 88 & 93 & 0.98 \\
\hline indistinct soft drusen & 118 & 50 & 68 & 84 & 86 & 0.02 \\
\hline distinct soft drusen & 184 & 13 & 41 & 54 & 59 & 0.002 \\
\hline $\begin{array}{l}\text { pigmentary } \\
\text { abnormality }\end{array}$ & 374 & 16 & 31 & 44 & 49 & $<0.001$ \\
\hline hyperpigmentation & 368 & 16 & 31 & 44 & 48 & $<0.001$ \\
\hline hypopigmentation & 262 & 14 & 29 & 29 & 33 & 0.14 \\
\hline
\end{tabular}

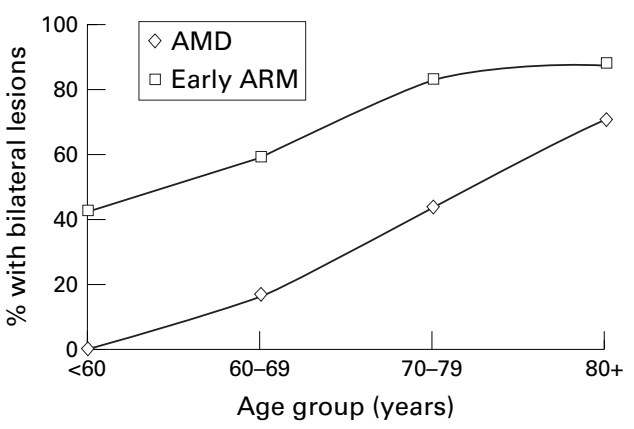

Figure 1 Influence of age on bilateral involvement by early and late lesions among people with signs of age related maculopathy.

EFFECT OF AGE AND SEX ON THE BILATERAL INVOLVEMENT OF LESIONS

As expected, a consistent age related increase in bilateral involvement for most lesions was found. This was highly significant using the Mantel-Haenzsel $\chi^{2}$ test for trend and logistic regression analyses, for almost all lesions (Table 2 and Fig 1). Exceptions were the bilateral prevalence of the following three lesions, two of which were relatively infrequent. Bilateral geographic atrophy was not observed until age 70-79 and declined for people aged 80 or older. Reticular drusen were nearly always found to be present in both eyes. Hypopigmentation was also uncommonly present in both eyes and showed no significant increase in bilateral involvement with increasing age. For all four soft drusen characteristics graded, a similar and statistically significant age related increase in bilateral involvement was found (Mantel-Haenszel $\chi^{2}$ for trend, $\mathrm{p}<0.001$, results not shown).

All age related maculopathy lesions were more frequently bilateral in women than men, apart from indistinct and distinct soft drusen. This consistent difference remained after adjusting for age, current smoking, and family history of AMD (Table 3). A significantly higher rate of bilateral involvement in women than men was found for neovascular AMD, odds ratio (OR) 7.7, 95\% confidence intervals (CI) 1.3-46.7. For all other lesions, nonsignificant increased odds ratios were found for bilateral involvement in women (OR 2.5 for any $A M D, O R 2.4$ for geographic atrophy, and OR 1.6 for early ARM). For individual or grouped retinal pigmentary abnormalities, non-significant odds ratios of 1.3:1.4 were found. All four soft drusen characteristics were also more frequently bilateral symmetry in females, with non-significant increased age adjusted odds ratios ranging from 1.2 to 1.4 (results not shown).

As current smoking and family history of AMD have been shown to have strong associations with $\mathrm{AMD},{ }^{18-20}{ }^{24-26}$ we also tried to assess the relation between $\mathrm{AMD}$ family history and bilateral involvement by ARM lesions. For the following lesions: (1) any ARM, (2) early ARM, (3) indistinct soft drusen and (4) reticular drusen, all cases reporting an AMD family history had the lesions present bilaterally. 
Table 3 Effect of sex on the frequency of bilateral involvement by age related macular degeneration (AMD) and age related maculopathy (ARM) lesions

\begin{tabular}{|c|c|c|c|c|c|}
\hline \multirow[b]{2}{*}{ Outcomes } & \multicolumn{2}{|c|}{ Female } & \multicolumn{2}{|l|}{ Male } & \multirow[b]{2}{*}{$\begin{array}{l}\text { Multivariate adjusted } \\
\text { odds ratio }(95 \% \mathrm{CI})^{*}\end{array}$} \\
\hline & $\begin{array}{l}\text { Total } \\
\text { cases }\end{array}$ & $\begin{array}{l}\text { No of } \\
\text { bilateral } \\
(\%)\end{array}$ & $\begin{array}{l}\text { Total } \\
\text { cases }\end{array}$ & $\begin{array}{l}\text { No of } \\
\text { bilateral } \\
(\%)\end{array}$ & \\
\hline Any ARM & 139 & $116(83)$ & 91 & $67(74)$ & $1.6(0.8-3.2) \dagger$ \\
\hline Any AMD & 47 & $30(64)$ & 22 & $9(41)$ & $2.5(0.8-8.0)$ \\
\hline neovascular AMD & 33 & $17(52)$ & 15 & $2(13)$ & $7.7(1.3-46.7)$ \\
\hline geographic atrophy & 25 & $16(64)$ & 14 & $6(43)$ & $2.4(0.6-10.0)$ \\
\hline Any early ARM & 92 & $74(80)$ & 69 & $49(71)$ & $1.6(0.7-3.5) \dagger$ \\
\hline $\begin{array}{l}\text { reticular drusen } \\
\text { indistinct soft }\end{array}$ & 25 & $24(96)$ & 10 & $8(80)$ & $8.5(0.5-133.1) \ddagger$ \\
\hline drusen & 63 & $50(79)$ & 55 & $43(78)$ & $1.0(0.4-2.6) \dagger$ \\
\hline $\begin{array}{l}\text { distinct soft drusen } \\
\text { pigmentary }\end{array}$ & 104 & $51(49)$ & 80 & $36(45)$ & $1.1(0.6-2.0)$ \\
\hline abnormality & 190 & $78(41)$ & 184 & $62(34)$ & $1.3(0.8-1.9)$ \\
\hline hyperpigmentation & 186 & $77(41)$ & 182 & $61(34)$ & $1.3(0.8-2.0)$ \\
\hline hypopigmentation & 141 & $44(31)$ & 121 & $29(24)$ & $1.4(0.8-2.5)$ \\
\hline
\end{tabular}

^Adjusted for age, family history of AMD and current smoking.

†Adjusted for age and current smoking only as no unilateral cases had a family history of AMD. $\ddagger$ Adjusted for age only as no unilateral cases had a family history of AMD or currently smoked.

\section{Discussion}

Although the prognosis of bilateral macular drusen has been reported from case series, ${ }^{13} 14$ few studies have examined the bilaterality of individual ARM lesions, ${ }^{12} 2728$ with no data from a population reported to date. Population based data on the bilateral frequency of ARM and individual ARM lesions is valuable as it is not affected by the selection biases inherent in clinic based samples. Accurate estimates of the frequency of bilateral involvement by ARM lesions would assist clinicians to predict the cost of AMD and to plan eye care and visual rehabilitative services for this disease.

In our study, any ARM lesion was found in both eyes of $80 \%$ of gradable subjects, with both eyes affected in $57 \%$ of the AMD cases. This proportion of bilateral AMD is only slightly higher than the $42 \%$ rate $(34 / 77)$ calculated from the Beaver Dam Eye Study report, ${ }^{29}$ and may be due to the older age profile of our study population. Our rates for any ARM are also comparable to the Framingham Eye Study report,${ }^{30}$ despite the use of different diagnostic criteria and methods of ascertainment. In Framingham, the bilateral proportion of "senile macular degeneration" cases was $64 \%, 71 \%$, and $79 \%$ for age groups $52-64$, 65-74, and 75-85, respectively. ${ }^{30}$

As the major determinant of ARM is increasing age,$^{21}{ }^{29-31}$ it could be expected that increasing bilateral involvement by individual ARM lesions would also be strongly age dependent. While this was found for most ARM lesions, we found no significant age related increase in bilateral involvement by hypopigmentation, a relatively common lesion. The contrasting bilaterality rates found for individual ARM lesions may imply that indistinct or reticular soft drusen are highly specific markers for ARM, while hypopigmentation may be a less specific manifestation of this disease. However, we can not exclude the possibility that measurement error or grading difficulty could have affected the grading of hypopigmentation or that some findings may be due to chance as a result of the multiple analyses performed.
In the Beaver Dam Eye Study population, women 75 years of age or older had a significantly higher frequency of neovascular $\mathrm{AMD}^{29}$ as well as a higher 5 year incidence rate of early ARM (OR 2.2, compared with men). ${ }^{32}$ In our study population, women had higher age specific prevalence rates for AMD lesions and soft drusen than men. ${ }^{21}$ Furthermore, 15 of the 16 people bilaterally blind from AMD were women, which is reflected in the very high age adjusted odds ratio (7.7) found for bilateral neovascular AMD in women compared with men in our study. For AMD and all individual ARM lesions apart from soft drusen, bilateral involvement was more frequent in women than men, after adjusting for age, current smoking, and AMD family history. An increased life expectancy for women could partly explain the different rate of bilateral AMD between women and men, but not the observed differences in risk for bilateral early ARM or pigmentary abnormalities. Hormonal factors in women could explain these consistent differences, as the Rotterdam study has reported an increased risk of AMD for early menopause in women. ${ }^{33} \mathrm{~A}$ decreased risk of neovascular AMD has also been reported for women taking postmenopausal oestrogen therapy. ${ }^{17}$

AMD family history was associated with bilateral involvement of most lesions and was absent in all unilateral cases of any ARM, early ARM, indistinct soft drusen, and reticular drusen. Strong familial aggregation of AMD was recent reported from the Rotterdam Eye Study. ${ }^{20}$ A recent case-control study also reported significant familial aggregation at a lower strength. ${ }^{19}$ Our finding is consistent with both these findings and further emphasises the role of genetic influences in ARM.

In summary, the Blue Mountains Eye Study has provided age specific bilateral prevalence rates for AMD and ARM in older Australians. After adjusting for the effects of age, current smoking, and AMD family history, the study suggests an increased risk of bilateral AMD and early ARM in women, statistically significant for bilateral neovascular AMD. An AMD family history may also predict bilateral involvement of most individual ARM lesions. This study was supported by the Australian Department of
Health, Housing and Community Services (RADGAC grant) Health, Housing and Community Services (RAD
and the Save Sight Institute, University of Sydney.

1 Cooper RL. Blind registrations in Western Australia: a five year study. Aust NZ $\mathcal{f}$ Ophthalmol 1989;107:875-9.

2 Attebo K, Mitchell P, Smith W. Visual acuity and the causes of visual loss in Australia. The Blue Mountains Eye Study.

3 Hyman L. Epidemiology of AMD. In: Hampton RG, Nelson PT, eds. Age-related macular degeneration. Principles and practice. New York: Raven Press, 1992:1-35.

4 Ghafour IM, Allan D, Foulds WS. Common causes of blindness and visual handicap in the west of Scotland. BrF Ophthalmol 1983;67:209-13.

5 Kahn HA, Moorhead HB. Statistics on blindness in the model reporting area, 1969-1970. 1973;HEW (NIH)73-427 (abstract).

6 The International AMD Epidemiological Study Group. An international classification and grading system for agerelated maculopathy and age-related macular degeneration. Surv Ophthalmol 1995;39:367-74.

7 Gregor Z, Bird AC, Chisholm IH. Senile disciform macular Gregor Z, Bird AC, Chisholm IH. Senile disciform macular
degeneration in the second eye. Br f Ophthalmol 1977;61:
$141-7$. 
8 Strahlman ER, Fine SL, Hillis A. The second eye of patients with senile macular degeneration. Arch Ophthalmol 1983; 101:1191-3.

9 Roy $M$, Kaiser Kupfer $M$. Second eye involvement in age-related macular degeneration: a four-year prospective study. Eye 1990;4:813-18.

10 Bressler SB, Maguire MG, Bressler NM, et al. Relationship of drusen and abnormalities of the retinal pigment epithelium to the prognosis of neovascular macular degeneration The Macular Photocoagulation Study Group. Arch Ophthalmol 1990;108:1442-7.

11 Macular Photocoagulation Study Group. Five-year follow-up of fellow eyes of patients with age-related macular degeneration and unilateral extrafoveal choroidal neovascularization. Macular Photocoagulation Study Group. Arch Ophthalmol 1993;111:1189-99.

12 Lavin MJ, Eldem B, Gregor ZJ. Symmetry of disciform scars in bilateral age-related macular degeneration. Brf Ophthalmol 1991;75:133-6.

13 Smiddy WE, Fine SL. Prognosis of patients with bilateral macular drusen. Ophthalmology 1984;91:271-7.

14 Holz FG, Wolfensberger TJ, Piguet B, et al. Bilateral macular drusen in age-related macular degeneration. Prognosis lar drusen in age-related macular degeneration. P
and risk factors. Ophthalmology 1994;101:1522-8.

15 Stur M, Tittl M, Reitner A, Meisinger V. Oral zinc and the second eye in age-related macular degeneration. Invest Ophthalmol Vis Sci 1996;37:1225-35.

16 Wang Q, Chappell RJ, Klein R, et al. Pattern of age-related maculopathy in the macular area. The Beaver Dam Eye Study. Invest Ophthalmol Vis Sci 1996;37:2234-42.

17 Eye Disease Case-Control Study Group. Risk factors for neovascular age-related macular degeneration. Arch Ophthalmol 1992;110:1701-8.

18 Smith W, Mitchell P, Leeder SR. Smoking and age-related maculopathy: the Blue Mountains Eye Study. Arch Ophthalmol 1996;114:1518-23.

19 Seddon JM, Ajani UA, Mitchell BD. Familial aggregation of age-related maculopathy. Am f Ophthalmol 1997;123:199206.

20 Klaver CCW, Wolfs RCW, van Duijn CM, et al. Familial aggregation of age-related macular degeneration in the
Rotterdam Study, Invest Ophthalmol Vis Sci 1997;38: abstract 4481

21 Mitchell P, Smith W, Attebo K, et al. Prevalence of age-related maculopathy in Australia: the Blue Mountains Eye Study. Ophthalmology 1995;102:1450-60.

22 Early Treatment Diabetic Retinopathy Study (ETDRS). Springfield, VA: ETDRS Coordinating Center, Department of Epidemiology and Preventive Medicine. National Technical Information Service, 1980.

23 Klein R, Davis M, Magli Y, et al. The Wisconsin age-related maculopathy grading system. Ophthalmology 1991;98: 1128-34.

24 Vingerling JR, Klaver CC, Hofman A, et al. Epidemiology of age-related maculopathy. Epidemiol Rev 1995;17:347-60.

25 Seddon JM, Willett WC, Speizer FE, et al. A prospective study of cigarette smoking and age-related macular degeneration in women. $\mathcal{F A M A} 1996 ; 276: 1141-6$.

26 Christen WG, Glynn RJ, Manson JE, et al. A prospective study of cigarette smoking and risk of age-related macular degeneration in men. $\mathcal{F A M A} 1996 ; 276: 1147-51$.

27 Pauleikhoff D, Wormald RP, Wright L, et al. Macular disease in an elderly population. Ger F Ophthalmol 1992;1: $12-15$.

28 Barondes M, Pauleikhoff D, Chisholm IC, et al. Bilaterality of drusen. Br f Ophthalmol 1990;74:180-2.

29 Klein R, Klein BE, Linton KL. Prevalence of age-related Klein R, Klein BE, Linton KL. Prevalence of age-related
maculopathy. The Beaver Dam Eye Study. Ophthalmology maculopathy. The

30 Sperduto RD, Seigel D. Senile lens and senile macular changes in a population-based sample. Am $\mathcal{F}$ Ophthalmol 1980;90:86-91.

31 Vingerling J, Dielemans I, Hofman A, et al. The prevalence of age-related maculopathy in the Rotterdam Study. Ophthalmology 1995;102:205-10.

32 Klein R, Klein BE, Jensen SC, et al. The five-year incidence and progression of age-related maculopathy: the Beaver Dam Eye Study. Ophthalmology 1997;104:7-21.

33 Vingerling JR, Dielemans I, Witteman JC, et al. Macular degeneration and early menopause: a case-control study. degeneration and early 